\title{
CMS luminosity precision in 2017 proton-proton collisions at LHC at $\sqrt{\mathrm{s}}=13 \mathrm{TeV}$
}

\author{
Christopher Palmer for the CMS collaboration* ${ }^{\dagger}$ \\ Princeton University / CMS Collaboration \\ E-mail: cp10eprinceton.edu
}

\begin{abstract}
The calibration for the luminosity measurement by the CMS experiment for the 2017 protonproton LHC run at $\sqrt{s}=13 \mathrm{TeV}$ is described. To guarantee smooth and uninterrupted luminosity measurements the CMS experiment is equipped in Run II with three online luminometers: the Forward Calorimeter (HF), the Pixel Luminosity Telescope (PLT), and the Fast Beam Condition Monitor (BCM1F). For the offline luminosity measurement and a cross check of the online detectors the pixel detector is used (Pixel Cluster Counting, PCC). The principal calibration is derived from the analysis of the Van der Meer scan program in CMS taken during LHC fill 6016. In addition, the performance and stability of the CMS luminometers are also evaluated using emittance scans taken throughout the course of the year. The systematic uncertainty in the absolute calibration from the Van der Meer scan is derived with a precision of $1.5 \%$, with the dominant contributions arising from the $x-y$ correlations of the beam shape and the scan to scan variation. The total systematic uncertainty, including terms from stability and linearity effects, is $2.3 \%$. For a low pile-up portion of data at the end of 2017 the calibration uncertainty was lower $(1.7 \%)$ because of lower impact from linearity uncertainty.
\end{abstract}

ICHEP 2018, XXXIX International Conference on High Energy Physics

4-11 July 2018

Seoul, Republic of Korea

* Speaker.

${ }^{\dagger}$ Many thanks to CMS collaborators who worked tirelessly on these precision measurements throughout 2017. 


\section{CMS Luminometers}

CMS has five detectors from which luminosity can be derived: the forward hadronic calorimeter (HF), the inner silicon pixel detector (pixel cluster counting, PCC), the Pixel Luminosity Telescope (PLT), the muon drift tubes (DT), and the Fast Beam Condition Monitor (BCM1F). The primary luminosity used for the 2017 measurement is from the HF. This is supplemented with PCC and PLT. DT and BCM1F were used primarily for cross check studies and online measurements.

The HF luminosity measurement uses a dedicated readout system installed in the HF calorimeter. The HF provided the primary online luminosity measurement for CMS during Run 1 of the LHC and has continued to provide excellent performance through Run 2. The algorithm used for 2017 uses the sum of the transverse energy ET (HFET).

The PCC method uses the rate of pixel clusters in the CMS pixel detector to provide a luminosity measurement. This method requires that portions of the pixel detector, which are not consistent in availability and response throughout the data-taking period, be excluded. The large area of the pixel detector and the relatively low occupancy provides a measurement with good statistical precision. The stability of the measurement over time is typically very good.

The PLT is a dedicated system for measuring luminosity using silicon pixel sensors, installed at the beginning of 2015 for Run 2. There are a total of 48 sensors arranged into 16 "telescopes", eight at either end of CMS outside the pixel endcap. Each telescope contains three sensor planes arranged nearly parallel to the beam pipe. The sensors measure $8 \times 8 \mathrm{~mm}$, divided into 80 rows and 52 columns, although only the central region of the sensors is used to reduce the contribution from background. PLT measures the rate of "triple coincidences", where a hit is observed in all three planes. This typically corresponds to a track from a particle originating at the interaction point. The overall mean rate for PLT (and also in BCM1F) is estimated using the zero-counting method.

Over the course of 2017, the PLT accumulated radiation damage in the sensors resulted in a loss of efficiency. The effects of this damage were compensated for by increasing the high voltage applied to the sensors; however, there were still several periods where the PLT had lower efficiency than normal. Corrections for these efficiency losses were measured with emittance scan data [2].

\section{Van der Meer Calibration Program and Results}

Most LHC luminometers derive their absolute calibrations from special scan programs where so-called Van der Meer (VdM) scans are performed. These scans are repeated numerous times in order to reduce statistical uncertainty and ensure reproducibility. Further scans are performed to derive beam positions during the scan and to account for assumptions made in the standard VdM analytic computation (e.g. the proton bunch density can be factorized in orthogonal transverse directions). This program is described in great detail in Ref. [1]. Results and important features are described.

The basic procedure of the VdM scan is to record luminometer rates in scan steps as two beams are separated with varying distance. This type of scan is performed twice-in two orthogonal, transverse directions. At each scan step the beams are held fixed long enough to obtain reasonable statistical precision on the rate of the luminometer. After the scans are completed, the rates are normalized with the product of the beam currents and plotted against the separation of the beams. 
This Gaussian-like distribution is fit to obtain the width of the beams' overlap and the peak value of the luminometer.

The limiting systematics of this analysis are based on the uncertainty of input data, the potential biases from assumptions, and uncertainties from various corrections. The three main inputs into these fits are: number of protons per beam, positions of the beams, and the rate of the luminometers. The number of protons per beam is generally normalized by LHC with a relatively small systematic uncertainty. However, the position of the beams need numerous checks and corrections. There are both systematic effects overall from LHC calibration errors and local position-dependent deviations from electro-magnetic effects that generally push the beams apart when they pass closely together (but not head-on). The length scale analysis corrects for the imperfections in LHC calibrations, and classical electromagnetism calculations correct for the beam-beam effects. In addition, this two one-dimensional fitting scheme is based on the factorization of the density of proton bunches functions into the product of two one-dimensional functions. This is not strictly true and a method of "beam imaging" has been developed in CMS to produce two-dimensional models with x-y correlation supplied in the model. The length scale corrections and $x-y$ correlation corrections will be described in detail while the rest of the VdM calibration corrections and systematics are listed in Table 1 and described in [1].

Table 1: Summary of the systematic uncertainties entering the VdM normalization for $\sqrt{s}=13 \mathrm{TeV}$ protonproton collisions.

\begin{tabular}{|l|c|c|}
\hline Systematic & Correction (\%) & Uncertainty (\%) \\
\hline Length scale & -0.9 & 0.3 \\
Orbit drift & - & 0.2 \\
$x-y$ correlations & +0.8 & 0.8 \\
Beam-beam deflection & +1.6 & 0.4 \\
Dynamic- $\beta^{*}$ & - & 0.5 \\
Beam current calibration & - & 0.3 \\
Ghosts and satellites & - & 0.1 \\
Scan to scan variation & - & 0.9 \\
Bunch to bunch variation & - & 0.1 \\
Cross-detector consistency & $+0.4--0.6$ & 0.6 \\
\hline Total normalization uncertainty & & 1.5 \\
\hline
\end{tabular}

\subsection{Length Scale Scans and Corrections}

Length scale scans are made in order to check that LHC is moving the beams by the amount reported. It is assumed that for each beam and in each transverse direction, there may be a correction needed. The location of CMS reconstructed proton-proton collisions are used as the standard for the correct scale. The average position of the reconstructed collisions is plotted against the position given by LHC for a comparison. In practice, because both beams are moved symmetrically during $\mathrm{VdM}$ scans, only one effective correction to beam separation is needed per transverse direction.

Two methods of deriving the length scale corrections are employed, compared and then combined. The "constant separation" method arrives only at the combined correction in both directions, while the "variable separation" method measures a correction for each beam and each transverse direction. Two datasets were taken for each type of scan and then analyzed independently. The results in each case are stunningly compatible. Figure 1 highlights the scans' results for one direction from each type of scan. The total systematic error in the $\mathrm{VdM}$ calibration from combining both of these methods is $0.3 \%$. 

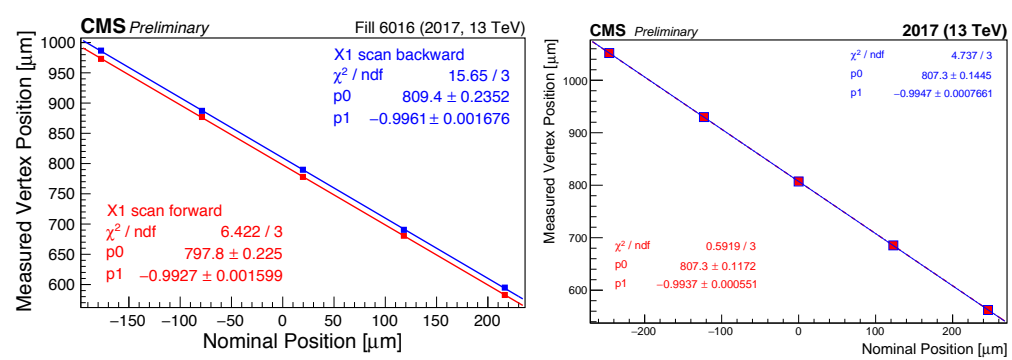

Figure 1: Left: reconstructed beam spot position as a function of the nominal beam separation in the constant separation scans in the $x$ plane. The forward (backward) scan is shown in red (blue). Right: Length scale calibration scan, using the variable separation procedure, for the $x$ direction of beam 1 in red and beam 2 in blue. Shown is the measured displacement of the luminous centroid as a function of the expected displacement based on the corrector bump amplitude. The line is a linear fit to the data. Errors are of statistical nature. The slopes of all four fits in both methods are highly compatible and results in essentially the same correction.

\subsection{XY correlation corrections with beam imaging scans}

Dedicated scans are made in which one beam is held fixed and the other beam is moved across the fixed beam in each of the orthogonal transverse directions. The resulting rate measurements provide a probe of the proton bunch densities in the fixed beam. The locations of reconstructed proton-proton collisions from each pair of beam imaging scans are fit using the two independent two-dimensional models convoluted with a proton-proton resolution model, which is essentially a smearing.

The two-dimensional models have correlation terms that can essentially rotate the $x$-y coordinates. The models are fit with correlation and the beam overlap is computed. In order to derive a correction for the measured cross section from the VdM scan, the fitted bunch proton densities are used to simulate VdM scans. The product $\Sigma_{x} \Sigma_{y}$ from the Monte Carlo simulation of the VdM scan method can then be compared to the value from a direct integration of the non-factorized bunch proton densities. This yields an estimate of the inaccuracy introduced by using the beam overlap area $\Sigma_{x} \Sigma_{y}$, which does not account for the $x-y$ correlations of the bunch proton densities. Multiple simulations are performed to derive the central value of the correction and its statistical uncertainty. Additionally, a Monte Carlo simulation of beam imaging scans is performed to estimate any possible bias from the fit method, and the resulting uncertainty is added to the statistical one.

\subsection{Calibration consistency results}

After the final calculation of the individual cross sections, the total integrated luminosity is computed for the periods in the VdM scan fill when no scans were taking place. Since these periods feature a nearly constant luminosity at very low pile-up, any effects due to non-linearity should be negligible and hence the measurements should be equal among the different luminometers. However, in fact, a small differences among the observed luminosities are present. In order to have all luminometers calibrated to the same central scale, all visible cross sections of the five independent luminometers are adjusted such that they all give an equal amount of integrated luminosity in the calibration data. Table 2 summarizes the rescaling applied for each luminometer. A 
systematic uncertainty of $0.6 \%$, the largest correction applied in the rescaling, as a measure of the associated uncertainty from the non-closure of $\mathrm{VdM}$ calibration on $\mathrm{VdM}$ fill data.

Table 2: Summary of measured integrated luminosity during stable periods in the VdM fill. The third column indicates the difference between the luminosity and the average; a correction equal to the inverse of this difference is then applied to rescale the visible cross section of each luminometer to the average.

\begin{tabular}{|c|c|c|}
\hline Luminometer & Integrated luminosity $\left(\mathrm{nb}^{-1}\right)$ & Difference from average \\
\hline HFET & 28.42 & $-0.6 \%$ \\
BCM1F & 28.45 & $-0.4 \%$ \\
PLT & 28.56 & - \\
HFOC & 28.67 & $+0.3 \%$ \\
PCC & 28.71 & $+0.5 \%$ \\
\hline
\end{tabular}

In addition, among the six scan pairs and five luminometers there were several notable variations in the resulting calibration constants. The largest variation among the six scans (from an individual luminometer) was taken as the uncertainty for scan-to-scan variation (0.9\%). There was also nearly negligible variation among the results per bunch crossing and an additional uncertainty of $0.1 \%$ was assessed.

\section{Afterglow corrections}

Two corrections are applied to the primary HFET and secondary PCC measurements to account for afterglow effects. "Type 1" corrections account for the signal from a hit spilling over into the next $25 \mathrm{~ns}$ bunch crossing after a colliding bunch, while "Type 2" effects account for an exponentially decaying afterglow for several bunches following a colliding bunch due to activation of the surrounding detector material. These effects are measured using data from empty bunches, which should thus have zero luminosity, and corrections are derived and applied to the raw luminosity. The afterglow corrections (combining Type 1 and Type 2) for HFET amount to approximately $4 \%$ in the bunch immediately following a colliding bunch and $0.5 \%$ in the next following bunch, with the corrections for subsequent bunches less than $0.1 \%$.

To assess the systematic uncertainty in the HFET corrections, the residual luminosity in the empty bunch crossings with corrections are analyzed. The resulting deviation from zero can be used to estimate the uncertainty. The residuals are plotted as a function of time in Figure 2. An envelope around the residuals is used to assess the $0.2 \%$ and $0.3 \%$ uncertainties for Type 1 and Type 2 corrections respectively.

\section{Detector Response Stability and Linearity Uncertainty}

In order to assess the stability and the consistency in linear response, a series of comparisons is performed between all pairs of detectors. Overall, HFET and PCC provided very consistent luminosity values over the course of the entire year. The HF was more extensively studied at the end of 2017, and therefore HF was selected as the primary luminometer for CMS' 2017 data. PCC provided a consistent, highly correlated dataset, whose correlation was used to set an upper limit on the stability of the HFET luminosity to the level of $0.5 \%$ based on the RMS of the luminosity ratios in roughly 6 minute chunks of data. 

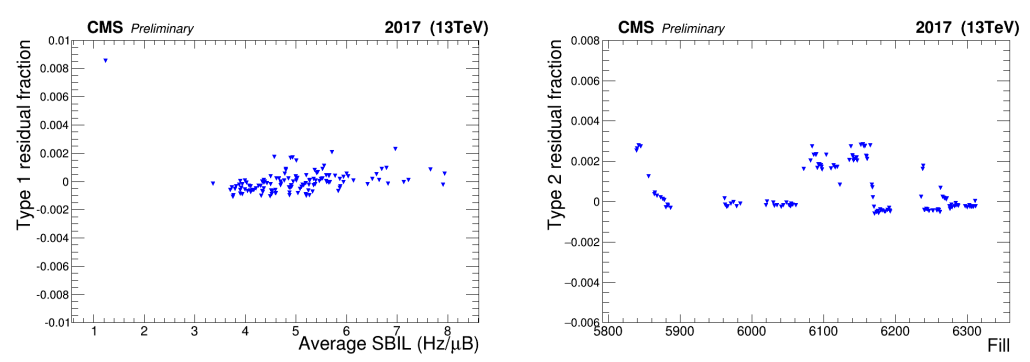

Figure 2: Left: HFET correction Type 1 residual fractions (remaining luminosity in empty bunches after corrections) as a function of average SBIL, after the non-linearity effect is corrected. Right: Type 2 residual fractions as a function of fill.

HFET has linearity corrections derived through the analysis of LHC emittance scan data [2]. PCC is expected to be linear from MC in absence of (or after corrections for) out-of-time pile-up (aka afterglow) corrections. All luminometer pairs were compared fill-by-fill and a slope was extracted. The mean and RMS of the slopes over the course of 2017 data were compared and the largest value was taken as the uncertainty on the linearity overall. This value was $0.3 \% /(\mathrm{Hz} / \mu \mathrm{B})$. A correction of the luminosity by this amount would result in a change of total integrated luminosity of $1.5 \%$. All stability uncertainties are summarized in Table 3. The total uncertainty on the integrated luminosity based on stability and linearity effects is $1.7 \%$.

Table 3: Summary of the systematic uncertainties entering the stability and extrapolation of $\sqrt{s}=13 \mathrm{TeV}$ proton-proton collisions.

\begin{tabular}{|l|c|}
\hline Systematic & Uncertainty (\%) \\
\hline Afterglow (HF) & $0.2 \oplus 0.3$ \\
Cross-detector stability & 0.5 \\
Linearity & 1.5 \\
CMS deadtime & 0.5 \\
\hline Total stability uncertainty & 1.7 \\
\hline
\end{tabular}

\section{Outlook}

The total uncertainty on the integrated luminosity of 2017 proton-proton collisions at $13 \mathrm{TeV}$ $(2.3 \%)$ is well balanced between calibrations and overall stability effects. In the future analysis of this data there is great potential to improve the overall precision. Among normalization uncertainties dynamic- $\beta^{*}$, scan to scan variation, and cross-detector consistency can be reduced considerably. Among stability systematics, the linearity uncertainty is the largest single uncertainty in the analysis and getting to a $0.5 \%$ level uncertainty is possible. With reductions among these uncertainties a total uncertainty of $1.5-1.7 \%$ is possible upon re-analysis of this dataset.

\section{References}

[1] CMS Collaboration, "CMS luminosity measurement for the 2017 data-taking period at $\sqrt{s}=13 \mathrm{TeV}$ ", CMS Physics Analysis Summary CMS-PAS-LUM-17-004, 2018.

[2] CMS Collaboration, "Emittance scans for CMS luminosity calibration in 2017", CMS Detector Report CMS-DP-2018-011, 2018. 